Article

\title{
Frailty Parameters, Morbidity and Mortality in Older Adults with Cancer: A Structural Equation Modelling Approach Based on the Fried Phenotype
}

\author{
Frederic Pamoukdjian ${ }^{1,2, *}$, Marie Laurent ${ }^{2,3}$, Claudia Martinez-Tapia ${ }^{2}$, Yves Rolland ${ }^{4,5}$, \\ Elena Paillaud $2,6,+\left(\mathbb{B}\right.$ and Florence Canoui-Poitrine ${ }^{2,7,+}$ \\ 1 APHP, Avicenne Hospital, Geriatric Department, Coordination Unit in Geriatric Oncology, \\ F-93000 Bobigny, France \\ 2 Univ Paris Est Creteil, INSERM, IMRB, F-94010 Creteil, France; marie.laurent@aphp.fr (M.L.); \\ claudia.martinez-tapia@aphp.fr (C.M.-T.); elena.paillaud@aphp.fr (E.P.); \\ florence.canoui-poitrine@aphp.fr (F.C.-P.) \\ 3 APHP, Henri-Mondor Hospital, Internal Medicine and Geriatric Department, Paris-Sud-Val-de-Marne \\ Geriatric Oncology Clinic, F-94000 Créteil, France \\ 4 Gérontopôle de Toulouse, Institut du Vieillissement, Centre Hospitalo-Universitaire de Toulouse, 37 Allée \\ Jules Guesde, 31000 Toulouse, France; rolland.y@chu-toulouse.fr \\ 5 UPS/INSERM UMR 1027, University of Toulouse III, Toulouse, France Faculté de Médecine, 37 Allées Jules \\ Guesde, 31000 Toulouse, France \\ 6 APHP, Paris Cancer Institute CARPEM, Geriatric Oncology Unit, European Georges Pompidou Hospital, \\ F-75015 Paris, France \\ 7 APHP, Henri-Mondor Hospital, Public Health Department, F-94000 Créteil, France \\ * Correspondence: frederic.pamoukdjian@aphp.fr; Tel.: +33-148-95-70-35; Fax: +33-148-95-70-36 \\ + These authors contributed equally to this work.
}

Received: 7 May 2020; Accepted: 9 June 2020; Published: 11 June 2020

\begin{abstract}
Background: to distinguish direct and indirect pathways to frailty phenotype, and quantify associations between two frailty components (i.e., sarcopenia and cachexia) regarding mortality and morbidity in older adults with cancer. Methods: all consecutive older outpatients with cancer were included in a prospective two-centre cohort study between 2013 and 2017 and had geriatric assessment. We used the frailty phenotype. Sarcopenia and cachexia were built as latent variables by including observed variables related to physical performances and related to nutrition and inflammation respectively. Structural equation modelling was used to distinguish between direct and indirect effects of the frailty parameters on the risk of death (Model 1) and the risk of morbidity (defined by unplanned hospitalization and/or disability and/or a fall; Model 2). The root mean square error of approximation (RMSEA) and the comparative fit index (CFI) were used to assess the model fit. Results: 603 older outpatients were included (mean age: $81.2 \pm 6.1$; women: $54 \%$; frailty phenotype: $58 \%$ ). The 6 -month mortality and morbidity rates were $18 \%$ and $64 \%$, respectively. The fit was good for both models (RMSEA and CFI $=0.029$ [0.017-0.039] and 0.99 for Model 1, and 0.028 [0.017-0.039] and 0.99 for Model 2, respectively). Sarcopenia and cachexia were both directly and significantly associated with 6-month mortality $\left(\beta^{\text {sarcopenia }}=0.18, p=0.01 ; \beta^{\text {cachexia }}=0.52, p<0.0001\right)$ and morbidity ( $\left.\beta^{\text {sarcopenia }}=0.37, p<0.0001 ; \beta^{\text {cachexia }}=0.19, p<0.02\right)$. Conclusions: sarcopenia and cachexia had a direct pathway with 6-month mortality and morbidity in older cancer patients.
\end{abstract}

Keywords: sarcopenia; cachexia; cancer; structural equation modelling; mortality; morbidity 


\section{Introduction}

In older adults, frailty is defined as a state of high vulnerability to stressors. It is associated with a number of poor health outcomes, including early mortality, disability, falls, and unplanned hospitalization [1]. Of the various indices of frailty developed over the last 20 years [2], one of the most frequently used for research purposes is Fried's frailty phenotype (based on five physical frailty criteria: shrinking, exhaustion, low physical activity, slowness, and weakness) [3], for which a pathophysiological model has been suggested [2].

Given the complexity of the pathophysiological links between the frailty phenotype's components, we reasoned that structural equation modelling (SEM) could usefully (i) distinguish direct and indirect pathways to frailty, and (ii) quantify associations between frailty components [4]. Thus, the frailty phenotype corresponds to a vicious circle centred on sarcopenia (due to comorbidities and pathological aging) which leads to exhaustion and a decrease in mobility (slowness), strength (weakness), physical activity, and overall energy expenditure-thus strengthening a chronic state of undernutrition. In turn, chronic undernutrition worsens the sarcopenia [3]. Sarcopenia is the age-associated loss of skeletal muscle mass, muscle strength and/or physical performances [5]. Cachexia is an ongoing loss of skeletal muscle mass that cannot be fully reversed by conventional nutritional support and that leads to progressive functional impairment [6].

Since cancer is a strong stressor and a prevalent disease in older adults, it is an appropriate pathological situation in which to characterize pathways leading to the frailty phenotype and the related poor health outcomes [7]. To the best of our knowledge, the pathophysiological model of the frailty phenotype was not validated in older adults with cancer.

Hence, we hypothesized that the Fried frailty phenotype is applicable to older patients with cancer, and that SEM could distinguish between the direct and indirect effects of frailty parameters, and especially sarcopenia and cachexia, on morbidity and mortality in this population. We sought to assess the plausibility of this pathophysiological model for explaining early death, disability, falls and unplanned hospitalization in older adults with cancer by using an SEM approach.

\section{Methods}

\subsection{Study Design and Population}

From 15 November 2013 to 30 September 2017, outpatient members of the French Physical Frailty in Elderly Cancer (PF-EC) cohort were screened for inclusion in the present study. The PF-EC prospective, observational, two-centre cohort study has been described in detail elsewhere [8]. Briefly, all consecutive older in- and outpatients referred for a geriatric assessment (GA) at two university hospitals in the greater Paris area were prospectively included in the PF-EC cohort once a diagnosis of cancer had been established, and before the cancer treatment decision had been made. For the purposes of the present study, we excluded inpatients because walking test data were not usually available for this subgroup (e.g., because infusions therapy prevented the performance of walking tests).

The inclusion date was considered to be the date of the patient's first consultation in the participating geriatric oncology department.

All participants provided their informed consent before inclusion in the study. The study was approved by the local independent ethics committee (Avicenne Hospital, Bobigny, France; reference: CLEA-2015-019).

\subsection{Cancer-Related and Demographic Data}

Demographic data (age and sex), cancer-related data (tumour site and extension: local, locally advanced, i.e., a non-resectable tumour with no distant metastases, or metastatic) were recorded at the first geriatric oncology consultation, as part of the geriatric assessment (GA). The type of treatment received by each patient was categorized as either supportive care alone or another type of care, and was recorded during the 6-month follow-up consultation. 


\subsection{Geriatric Assessment (GA)}

The GA was performed during the patient's first consultation in the geriatric oncology department. Comorbidities were assessed using the Cumulative Illness Rating Scale for Geriatrics (CIRS(G)). Total comorbidity burden was defined as a total CIRS(G) score above the median value of 14 [9]. Dependency was defined as a six-item activities of daily living (ADL) score below or equal to 5 out of 6 , and/or by a four-item simplified instrumental ADL score (IADL: using the telephone, transport, medications, and money management) of less than 4 out of $4[10,11]$. Malnutrition was defined as a body mass index (BMI) below $21 \mathrm{~kg} / \mathrm{m}^{2}$ [12]. Impaired mobility was defined as a short physical performance battery (SPPB) score below 9 out of 12 and/or a one-leg stance balance test time below $5 \mathrm{~s}$ [13,14]. Depressed mood was defined as a Mini-Geriatric Depression Scale (Mini-GDS) score of 1 or more out of 4 [15]. Cognitive impairment was defined as a Mini-Mental State Examination (MMSE) score below 24 out of 30 [16].

\subsection{Definition of Frailty}

Data on the frailty phenotype were collected during the patient's GA $[3,17]$. Frailty was defined as at least three of five criteria developed by Fried et al.

Shrinking was defined by an unintentional loss weight of at least $5 \%$ in the previous year.

Self-report of exhaustion (i.e., poor endurance and energy) was identified by two questions: "I felt that everything I did was an effort" and "I could not get going". Patients were asked "How often in the last week did you feel this way?" 0 = rarely or none of the time; 1 = some or a little of the time; $2=$ a moderate amount of the time; or $3=$ most of the time. Exhaustion was defined as an answer of " 2 " or " 3 " to either of these questions.

Low physical activity was defined by a single question. Individuals who denied doing daily leisure activities (such as walking or gardening) and/or sports activity at least once a week were categorized as physically inactive [18].

Slowness was defined as a slow gait speed, according to the sex- and height-adjusted cut-off values established by Fried et al. Gait speed (in $\mathrm{m} / \mathrm{s}$ ) was measured with a stopwatch for participants walking over a short distance $(4 \mathrm{~m}$, along a corridor) at their usual pace.

Weakness was defined as an impairment in maximum hand grip strength (in kilograms), according to the sex- and BMI-adjusted cut-off values established by Fried et al. Hand grip strength was measured twice for each hand using a hand-held dynamometer (EH101, Camry Electronic Ltd., Zhaoqing, China).

\section{Covariates}

Negative protein balance was assessed as the serum albumin level (measured in an immunoturbidimetric assay) over the 3 weeks immediately after the GA. A value below $35 \mathrm{~g} / \mathrm{L}$ was considered to be abnormal [12]. Inflammation was assessed in terms of the serum C-reactive protein (CRP) level (measured in an immunoturbidimetric assay) over the 3 weeks immediately after the GA. A value above $10 \mathrm{mg} / \mathrm{L}$ was considered to be abnormal [19].

\subsection{Adverse Health Outcomes}

Each patient had a follow-up consultation every three months during the first year after inclusion.

The main outcome was mortality in the 6 months after the GA. Vital status was determined by telephoning the patients or their family or by extracting data from medical records.

The secondary outcome was morbidity in the 6 months after the GA. This was a composite outcome defined as at least one of the following frailty-related events: the first unplanned hospitalization and/or disability (defined by the loss of at least 1 point on the ADL and/or IADL scales), and/or a fall. 


\subsection{Prespecified Structural Equation Modelling (SEM) of the Frailty Phenotype}

Structural equation modelling is based on the analysis of both observed and non-observed (i.e., latent) variables or constructs. The analysis uses a combination of correlation measures and regressions to assess the direct and indirect pathways within a pre-specified pathophysiological model [20]. Figure 1 shows the pre-specified structural equation modelling for the pathophysiology of the frailty phenotype adapted from Fried et al.'s theoretical model.

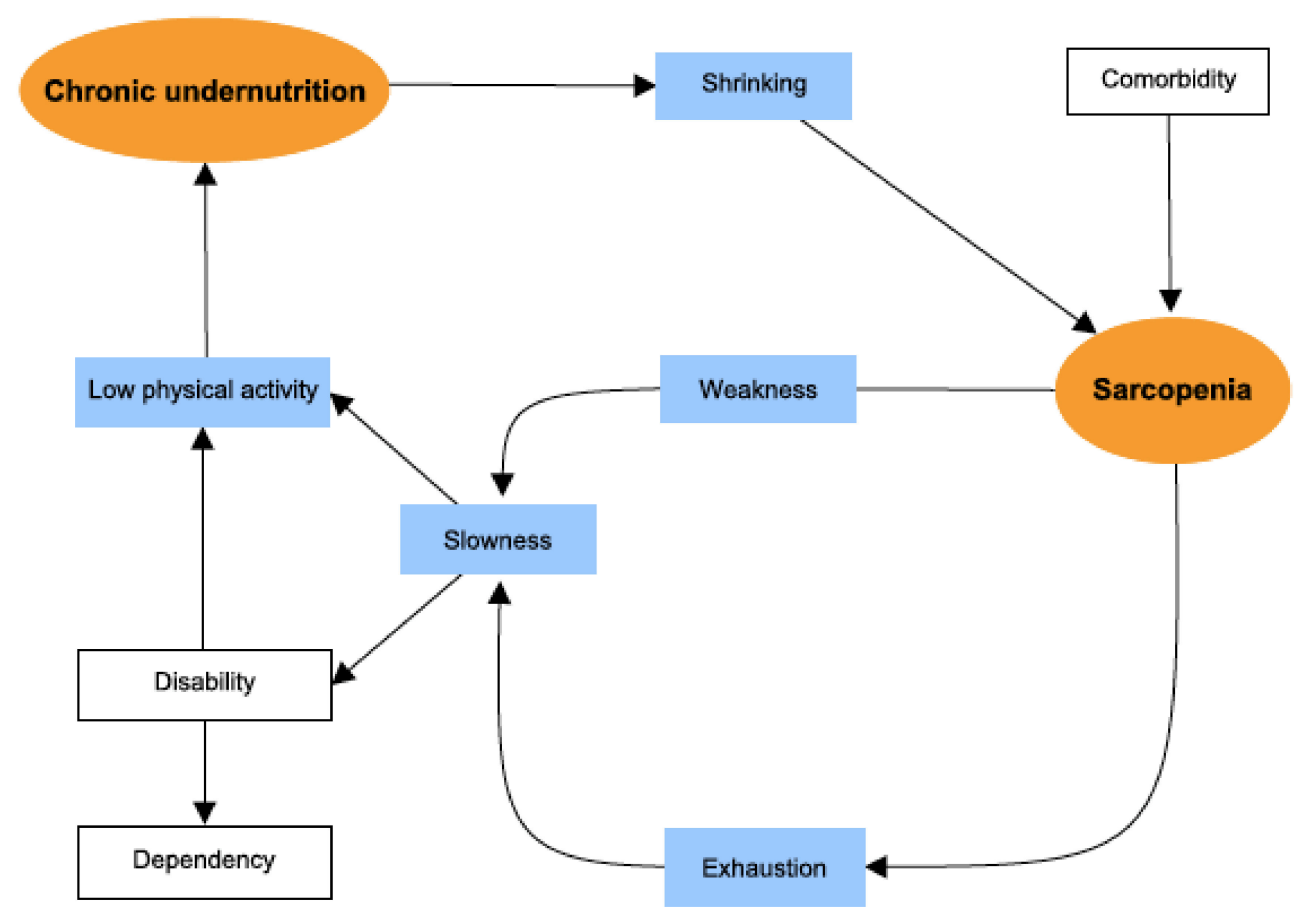

Figure 1. Pre-specified structural equation modelling (SEM) of the pathophysiology cycle for frailty. By convention, circles (orange) represent latent variables and rectangles represent observed variables. Blue rectangle $=$ Frailty criteria. The direction of the arrow indicates the hypothetical pathway, as proposed by Fried et al.

The central factor in the pathophysiological model is sarcopenia-the age-associated loss of skeletal muscle mass (quantitative impairment), muscle strength and/or physical performance (qualitative impairment) [5]. Due to the absence of a direct measurement of muscle mass in our study, sarcopenia was defined as a latent variable by including observed variables related to physical performances. Thus, we used low physical activity, slowness, weakness, one-leg stance balance test, and the SPPB score to define sarcopenia. For application to our population of patients with cancer, chronic undernutrition was used as a proxy for cachexia. The latter syndrome is defined as an ongoing loss of skeletal muscle mass that cannot be fully reversed by conventional nutritional support and that leads to progressive functional impairment. The pathophysiology of cachexia is characterized by a negative protein and energy balance (i.e., undernutrition) driven by a variable combination of reduced food intake and excessive catabolism (i.e., inflammation) [6]. Again, due to the absence of a direct measurement of muscle mass in our study, cachexia was defined as a latent variable by including observed variables related to nutrition and inflammation (shrinking, BMI, serum albumin level, and serum CRP level). Exhaustion was not used to build the latent variables because we suspected that it significantly influenced the relationship between cachexia and sarcopenia. This is in line with the pathophysiological model provided by Fried et al., in which sarcopenia induced exhaustion. The other observed variables were considered for adjustment of the structural equation model of the frailty phenotype. 
With the exception of the cancer site and cancer extension, each observed variable was binary (yes/no, with cut-off values defined above).

\subsection{Statistical Analyses}

Descriptive analysis: categorical variables were summarized as the number (percentage), and continuous variables were summarized as the mean \pm standard deviation (SD) or the median [interquartile range (IQR)].

Correlations: we first built a correlation matrix for each observed variable by using Cramer's V test (as is appropriate for a correlation between two qualitative variables).

Confirmatory factor analysis (CFA) [20]: the two latent variables were included in a CFA. The "diagonally weighted least squares" estimator was used to obtain estimates with standard errors and standardized coefficients.

Structural equation modelling [20]: the relationships between latent variables and endogenous variables were expressed as standardized coefficients (equivalent to the correlation coefficient). Linear or logistic regression was used to assess direct and indirect interactions between the two latent variables, as appropriate. Using a multivariate logistic regression analysis, two structural equation models (Models 1 and 2) were finally fitted to estimate the total direct effects of several predictors (i.e., sarcopenia + cancer cachexia + cancer site + cancer extension) for the two outcomes assessed (i.e., Model 1 for mortality, and Model 2 for morbidity). The result of the regression was expressed as the odds ratio (OR) by exponentiation of the standardized coefficients (equivalent to the $\beta$ coefficients).

Model fit [20]: we used two indices to characterize the quality of the CFA and the structural equation models. Firstly, the root mean square error of approximation (RMSEA) and its confidence interval (CI) describe the complexity of the model assessed. An RMSEA $<0.05$ was considered to be adequate. Secondly, the comparative fit index (CFI) compares the tested models with an independent model. A CFI $>0.95$ was considered to be adequate.

All tests were two-sided, and the threshold for statistical significance was set to $p<0.05$. The data were analysed using R statistical software (version 3.4.3, R Foundation for Statistical Computing, Vienna, Austria, http://www.R-project.org). Multivariate imputation by chained equations was used to handle missing data for shrinking $(n=9)$, exhaustion $(n=1)$, low physical activity $(n=1)$, slowness $(n=3)$, weakness $(n=8)$, BMI $(n=3)$, SPPB $(n=2)$, one-leg stance balance $(n=1)$, serum CRP $(n=112)$, and serum albumin $(n=100)$ via the MICE package in R. Structural equation modelling was performed by using the Lavaan R package.

\section{Results}

\subsection{Patients}

Of the 959 consecutive older (aged 65 and over) patients with cancer in the PF-EC cohort having been referred for a GA up until 30 September 2017, 356 were excluded because they were inpatients. Hence, 603 outpatients were included in the present study (Figure 2). 
The PF-EC cohort study:

Older adults with cancer $\geq 65$ years

referred for a Geriatric Assessment

before a therapeutic decision up to

September 30, 2017

$N=959$

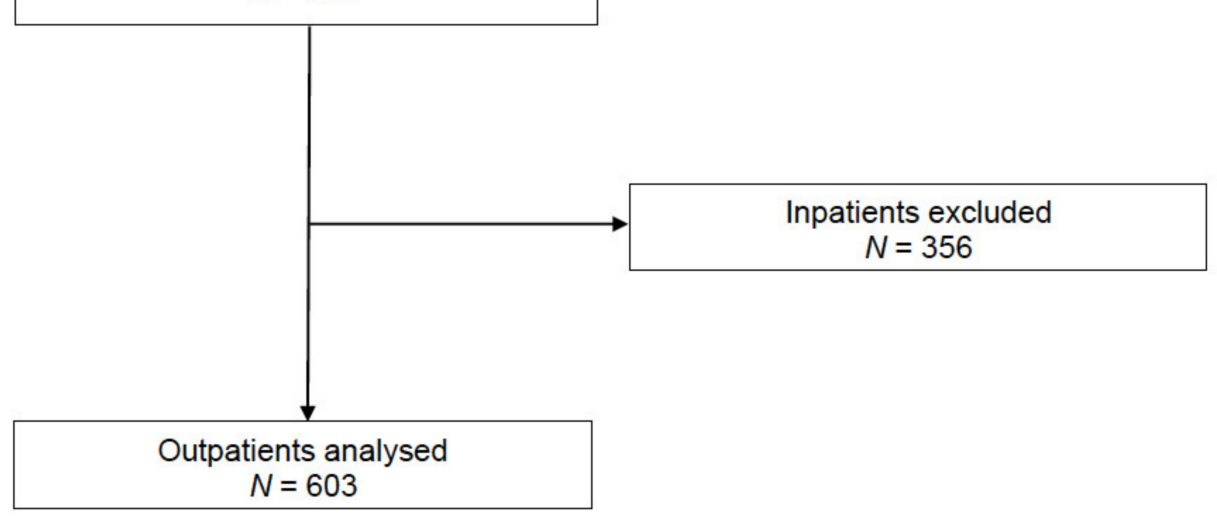

Figure 2. Flow chart.

\subsection{Baseline Characteristics of Patients and the Frailty Phenotype}

Table 1 shows the baseline characteristics of the included patients. The mean \pm SD age of the study population was $81.2 \pm 6.1$ years. Most of the patients were women $(54 \%)$, with solid tumours $(94 \%)$ and locally advanced (38\%) or metastatic cancer (45\%). Colorectal and breast cancers were the two most common types. The majority of the patients were frail. Figure 3 shows the proportion of patients with frailty criteria in the subgroup of frail patients: exhaustion $(36 \%)$, shrinking $(66.9 \%)$, slowness $(75.9 \%)$, weakness $(91.9 \%)$, and low physical activity $(99.7 \%)$. The proportion of patients with impairments in the GA varied from $14 \%$ to $64 \%$, depending on the domain and the thresholds used.

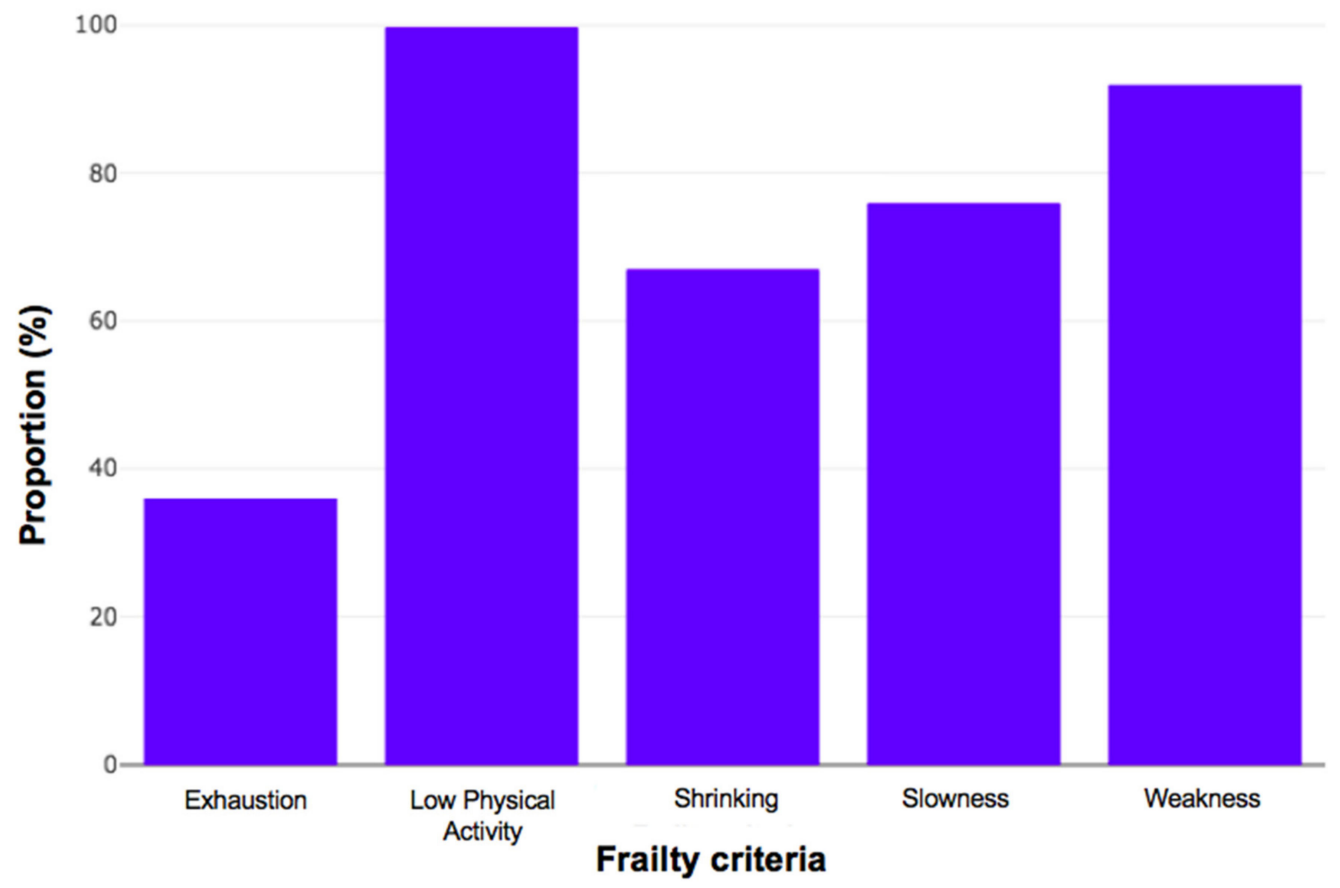

Figure 3. Proportion of frailty criteria in 352 frail older patients with cancer. 
Table 1. Baseline characteristics of the study population $(n=603)$.

\begin{tabular}{|c|c|}
\hline Variables & $n(\%)$ or Median [IQR] \\
\hline Age (year) & 82 [77-86] \\
\hline Sex (female) & $323(54)$ \\
\hline Frailty phenotype (yes) & $352(58)$ \\
\hline \multicolumn{2}{|l|}{ Cancer site } \\
\hline Colorectal & $109(18)$ \\
\hline Breast & $105(17)$ \\
\hline Lung & $92(15)$ \\
\hline Liver & $85(14)$ \\
\hline Digestive tract other than colorectal ${ }^{\text {a }}$ & $79(13)$ \\
\hline Genito-urinary tract & $40(7)$ \\
\hline Haematological malignancies & $34(6)$ \\
\hline Skin with melanoma & $16(2.5)$ \\
\hline Prostatic & $16(2.5)$ \\
\hline Others ${ }^{b}$ & $27(5)$ \\
\hline \multicolumn{2}{|l|}{ Cancer extension } \\
\hline Local & $104(17)$ \\
\hline Locally advanced & $228(38)$ \\
\hline Metastatic & $271(45)$ \\
\hline Supportive care only (yes) & $124(20.5)$ \\
\hline \multicolumn{2}{|l|}{ Comorbidities (CIRS(G)): } \\
\hline Total $>14$ & $269(45)$ \\
\hline \multicolumn{2}{|l|}{ Dependence } \\
\hline ADL $£ 5 / 6$ & $204(34)$ \\
\hline IADL $£ 3 / 4$ & $386(64)$ \\
\hline \multicolumn{2}{|l|}{ Nutrition } \\
\hline $\mathrm{BMI}<21 \mathrm{~kg} / \mathrm{m}^{2}$ & $84(14)$ \\
\hline Serum albumin level < $35 \mathrm{~g} / \mathrm{L}$ & $221(37)$ \\
\hline Serum $C R P \geq 10 \mathrm{mg} / \mathrm{L}$ & $267(44)$ \\
\hline \multicolumn{2}{|l|}{ Mobility } \\
\hline $\mathrm{SPPB}<9 / 12$ & $314(52)$ \\
\hline One-leg stance balance $<5 \mathrm{~s}$ & $448(74)$ \\
\hline \multicolumn{2}{|l|}{ Mood } \\
\hline Mini-GDS $\geq 1 / 4$ & $261(44)$ \\
\hline Missing data & $6(1)$ \\
\hline \multicolumn{2}{|l|}{ Cognition } \\
\hline MMSE $<24 / 30$ & $217(51)$ \\
\hline Missing data & $174(29)$ \\
\hline
\end{tabular}

a: pancreas $(n=28)$, gastric $(n=20)$, bile-duct $(n=15)$, oesophagus $(n=9)$, gastrointestinal stroma tumour $(n=4)$, anal $(n=3){ }^{b}$ : unknown primary site $(n=10)$, mesothelioma $(n=8)$, sarcoma $(n=5)$, head and neck $(n=3)$, thymoma $(n=1)$. ADL: activities of daily living; BMI: body mass index; CIRS(G): Cumulative Illness Rating Scale for Geriatrics; CRP: C-reactive protein; ECOG-PS: Eastern Cooperative Oncology Group performance status; GS: gait speed; IADL: instrumental activities of daily living; Mini-GDS: Mini-Geriatric Depression Scale; MMSE: Mini-Mental State Examination; SPPB: short physical performance battery.

\subsection{Correlations between Frailty Criteria and the Other Observed Variables}

Table 2 shows the correlation matrix for the observed qualitative variables. Shrinking was positively correlated with exhaustion, BMI, serum albumin, and serum CRP. Low physical activity was positively correlated with slowness, weakness, the one-leg stance balance and the SPPB score. Exhaustion was positively correlated with slowness and the SPPB score. The correlations were consistent with the pre-specified latent variables.

\subsection{Goodness of Fit}

In a CFA, we first tested the adequacy of the two latent variables (sarcopenia and cachexia). The model's fit was good, with an RMSEA (CI) of $0.032(0.012-0.049)$ and a CFI of 0.99 . There was significant covariance between the latent variables (standardized coefficient: $0.32 ; p<0.0001$ ). When we added exhaustion to each latent variable, the model's fit worsened: the RMSEA (CI) and a CFI were respectively $0.054(0.041-0.067)$ and 0.98 for sarcopenia; and $0.056(0.043-0.069)$ and 0.98 for cachexia. Thus, we chose to keep exhaustion as an exogenous mediating variable for the two latent variables. 
Table 2. Correlation matrix for observed variables.

\begin{tabular}{|c|c|c|c|c|c|c|c|c|c|c|c|c|c|c|c|}
\hline & $\begin{array}{c}\text { Shrinking } \\
\text { (Yes) }\end{array}$ & $\begin{array}{l}\text { Exhaustion } \\
\text { (Yes) }\end{array}$ & $\begin{array}{c}\text { Low } \\
\text { Physical } \\
\text { Activity } \\
\text { (Yes) }\end{array}$ & $\begin{array}{l}\text { Slowness } \\
\text { (Yes) }\end{array}$ & $\begin{array}{c}\text { Weakness } \\
\text { (Yes) }\end{array}$ & $\begin{array}{c}\text { One-Leg } \\
\text { Stance < } 5 \\
\text { s (Yes) }\end{array}$ & $\begin{array}{c}\text { SPPB }< \\
9 / 12 \\
\text { (Yes) }\end{array}$ & $\begin{array}{c}\text { BMI <21 } \\
\mathrm{kg} / \mathrm{m}^{2} \\
\text { (Yes) }\end{array}$ & $\begin{array}{l}\text { Albumin } \\
<35 \mathrm{~g} / \mathrm{L} \\
\text { (Yes) }\end{array}$ & $\begin{array}{c}\mathrm{CRP} \geq 10 \\
\mathrm{mg} / \mathrm{L} \text { (Yes) }\end{array}$ & $\begin{array}{c}\text { Age } \geq 82 \\
\text { Years (Yes) }\end{array}$ & $\begin{array}{l}\text { CIRS(G) } \\
\text { Total > } \\
14 \text { (Yes) }\end{array}$ & $\begin{array}{l}\text { Cancer } \\
\text { Site }\end{array}$ & $\begin{array}{c}\text { Cancer } \\
\text { Extension }\end{array}$ & $\begin{array}{l}\text { Supportive } \\
\text { Care Only } \\
\text { (Yes) }\end{array}$ \\
\hline Shrinking (yes) & 1 & & & & & & & & & & & & & & \\
\hline Exhaustion (yes) & 0.24 * & 1 & & & & & & & & & & & & & \\
\hline $\begin{array}{l}\text { Low physical } \\
\text { Activity (yes) }\end{array}$ & $0.13^{*}$ & 0.13 * & 1 & & & & & & & & & & & & \\
\hline Slowness (yes) & 0.10 * & 0.31 * & 0.27 * & 1 & & & & & & & & & & & \\
\hline Weakness (yes) & 0.06 & $0.18^{*}$ & 0.23 * & 0.34 * & 1 & & & & & & & & & & \\
\hline $\begin{array}{c}\text { One-leg stance }<5 \mathrm{~s} \\
\text { (yes) }\end{array}$ & 0.06 & $0.15^{*}$ & 0.33 * & $0.37 *$ & $0.35^{*}$ & 1 & & & & & & & & & \\
\hline SPPB < 9/12 (yes) & $0.09 *$ & $0.29 *$ & $0.29 *$ & 0.75 * & 0.38 * & 0.50 * & 1 & & & & & & & & \\
\hline BMI $<21 \mathrm{~kg} / \mathrm{m}^{2}$ (yes) & $0.18^{*}$ & $0.18^{*}$ & 0.04 & 0.05 & 0.03 & 0.06 & 0.03 & 1 & & & & & & & \\
\hline $\begin{array}{l}\text { Serum albumin }<35 \\
\mathrm{~g} / \mathrm{L} \text { (yes) }\end{array}$ & $0.22 *$ & 0.20 * & 0.10 * & 0.21 * & $0.14^{*}$ & 0.13 * & 0.20 * & $0.17^{*}$ & 1 & & & & & & \\
\hline $\begin{array}{l}\text { Serum } C R P \geq 10 \\
\text { mg/L (yes) }\end{array}$ & $0.20 *$ & $0.12 *$ & $0.08^{*}$ & 0.05 & 0.05 & 0.06 & 0.06 & $0.09 *$ & $0.30 *$ & 1 & & & & & \\
\hline Age $\geq 82$ years (yes) & 0.06 & 0.05 & $0.08^{*}$ & $0.16^{*}$ & $0.15^{*}$ & $0.20 *$ & $0.24^{*}$ & $0.09 *$ & 0.04 & 0.003 & 1 & & & & \\
\hline $\begin{array}{c}\text { CIRS(G) total > } 14 \\
\text { (yes) }\end{array}$ & 0.01 & 0.05 & $0.11 *$ & $0.25 *$ & $0.15 *$ & $0.19 *$ & $0.25 *$ & 0.11 * & 0.11 * & 0.10 * & $0.14^{*}$ & 1 & & & \\
\hline $\begin{array}{c}\text { Cancer site } \\
\text { Cancer extension }\end{array}$ & $0.30 *$ & 0.12 & 0.15 & $0.21 *$ & 0.14 & 0.14 & $0.20 *$ & 0.15 & $0.20 *$ & $0.25 *$ & $0.19^{*}$ & 0.12 & 1 & & \\
\hline Cancer extension & 0.08 & 0.05 & 0.02 & 0.02 & 0.02 & 0.04 & 0.02 & 0.02 & 0.04 & $0.15^{*}$ & 0.09 & 0.02 & 0.24 * & 1 & \\
\hline $\begin{array}{l}\text { Supportive care only } \\
\text { (yes) }\end{array}$ & 0.03 & $0.15^{*}$ & $0.08^{*}$ & $0.25 *$ & $0.17^{*}$ & $0.14^{*}$ & $0.23 *$ & 0.09 * & $0.13^{*}$ & 0.05 & $0.14^{*}$ & $0.09 *$ & $0.23 *$ & 0.08 & 1 \\
\hline
\end{tabular}

* $p$ value < 0.05 (Cramer's V test). SPPB: short physical performance battery; BMI: body mass index; CRP: C-reactive protein; CIRS(G) = Cumulative Illness Rating Scale for Geriatrics. 


\subsection{Pathways of the Frailty Phenotype Leading to Mortality}

The mortality rate (95\% confidence interval (CI)) 6 months after the initial GA was $17.9 \%$ (14.9-21.2) ( $n=108$ out of 603 ). The structural equation modelling of the relationship between frailty and mortality had a good fit: the RMSEA was $0.029(0.017-0.039)$, and the CFI was 0.99. The path diagram for the structural equation model 1 is summarized in Figure 4 . The total comorbidity burden was significantly associated with sarcopenia, while age and sex were not (data not included in Figure 4). While there was no direct association between the latent variables sarcopenia and cachexia, both were significantly linked to exhaustion. Sarcopenia and cachexia were both associated (independently of the cancer site and extension) with 6-month mortality in older outpatients with cancer. Cachexia had the strongest association with mortality. The provision of supportive care was not associated with mortality but was significantly linked to the sarcopenia variable. The path coefficients for regressions in the SEM are detailed in Table 3.

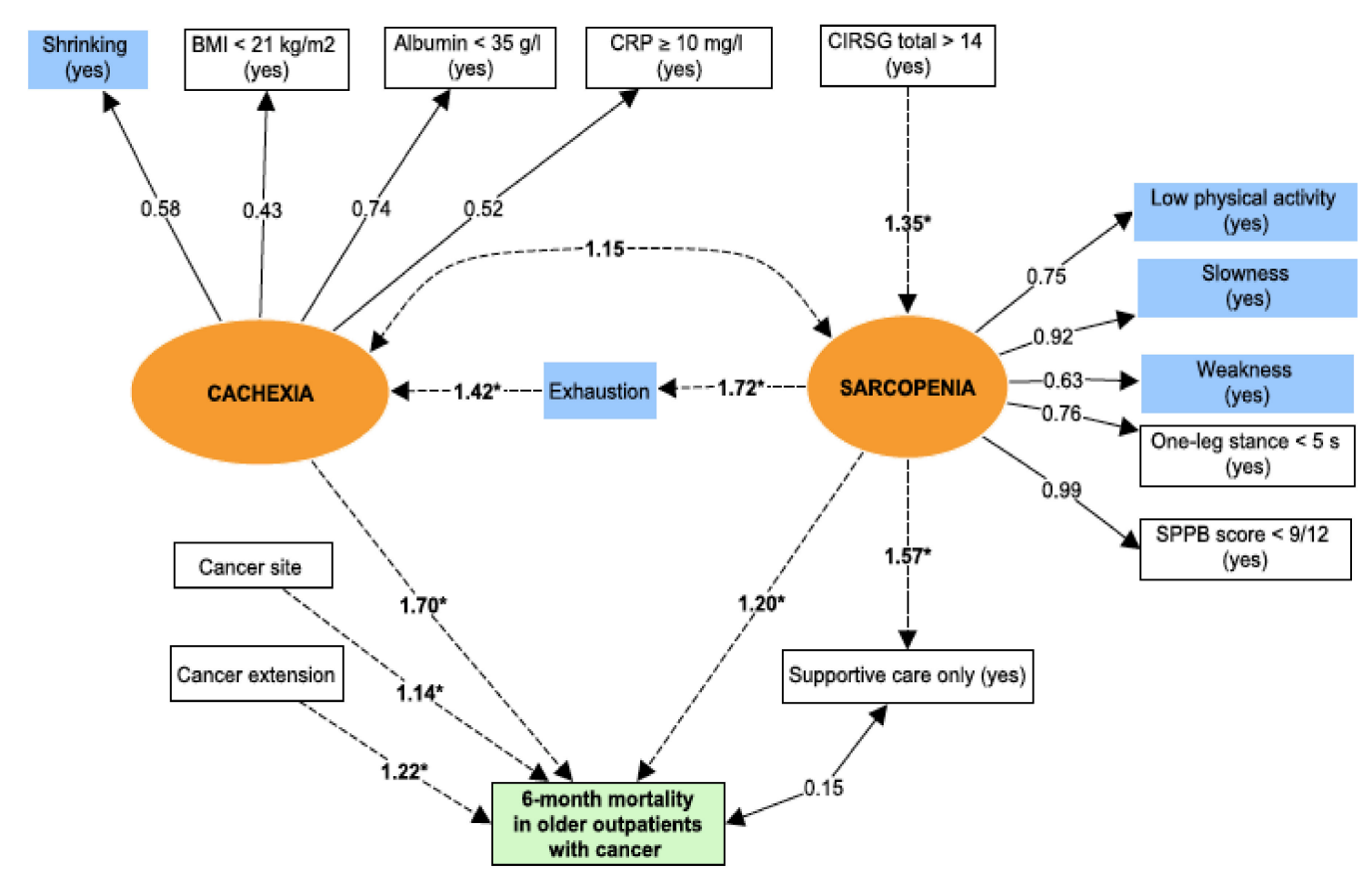

Figure 4. The pathways of the frailty phenotype leading to mortality. Circles (orange) = latent variables; Rectangles = observed variables; Correlations: dotted lines; Regressions: solid lines; Covariance: ${ }^{*} p$ value (for regressions) $<0.05$; Blue rectangle $=$ Frailty criteria; Green rectangle $=$ Outcome. Latent variables represent non-observed variables but which summarize a set of directly observed variables. Latent variables are constructed by using strong correlations between observed variables. Regressions are then used to assess association between latent variables and outcomes.

\subsection{Frailty Phenotype Pathways Leading to Morbidity}

The morbidity rate [95\% CI] 6 months after the initial GA was $64 \%$ (60-68) ( $n=386$ out of 603 ). Unplanned hospitalization affected $24 \%(n=146 / 603)$ of the patients, disability affected $48 \%(n=289$ out of 603); and falls affected $18.5 \%$ ( $n=112$ out of 603 ). Again, the fit for the structural equation modelling of the relationship between frailty and morbidity was good: the RMSEA was $0.028(0.017-0.039)$, and the CFI was 0.99. The path diagram for the structural equation model 2 is summarized in Figure 5. We obtained substantially the same results as for Model 1 (Table 3). Here, sarcopenia had the strongest association with morbidity. Cancer extension was not independently associated with morbidity. 
Table 3. Path coefficients estimated using regression (linear or logistic) in a structural equation model of the relationship between frailty components, mortality and morbidity in older outpatients with cancer.

\begin{tabular}{|c|c|c|c|c|}
\hline Models & Estimate & Standard Error & $\begin{array}{c}\text { Standardized Coefficient } \\
(\beta \text { Coefficient })\end{array}$ & $p$ Value \\
\hline \multicolumn{5}{|c|}{ Model 1} \\
\hline Sarcopenia $\sim$ CIRS(G) total > 14 & 0.64 & 0.1 & 0.30 & $<0.0001$ \\
\hline Exhaustion sarcopenia & 0.47 & 0.06 & 0.47 & $<0.0001$ \\
\hline Cachexia exhaustion & 0.16 & 0.05 & 0.37 & 0.001 \\
\hline \multicolumn{5}{|l|}{ Mortality* } \\
\hline$\sim$ sarcopenia & 0.18 & 0.07 & 0.18 & 0.01 \\
\hline$\sim$ cachexia & 1.30 & 0.29 & 0.52 & $<0.0001$ \\
\hline$\sim$ cancer site & 0.05 & 0.02 & 0.13 & 0.03 \\
\hline$\sim$ cancer extension & 0.23 & 0.07 & 0.20 & $<0.0001$ \\
\hline Supportive care only $\sim$ sarcopenia & 0.44 & 0.06 & 0.45 & $<0.0001$ \\
\hline \multicolumn{5}{|c|}{ Model 2} \\
\hline Sarcopenia $\sim$ CIRS(G) total $>14$ & 0.64 & 0.1 & 0.30 & $<0.0001$ \\
\hline Exhaustion sarcopenia & 0.47 & 0.06 & 0.48 & $<0.0001$ \\
\hline Cachexia $\sim$ exhaustion & 0.17 & 0.05 & 0.41 & $<0.0001$ \\
\hline \multicolumn{5}{|l|}{ Morbidity * } \\
\hline$\sim$ sarcopenia & 0.36 & 0.06 & 0.37 & $<0.0001$ \\
\hline$\sim$ cachexia & 0.46 & 0.20 & 0.19 & 0.02 \\
\hline$\sim$ cancer site & 0.05 & 0.02 & 0.14 & 0.01 \\
\hline$\sim$ cancer extension & 0.06 & 0.06 & 0.06 & 0.28 \\
\hline Supportive care only $\sim$ sarcopenia & 0.44 & 0.06 & 0.45 & $<0.0001$ \\
\hline
\end{tabular}

${ }^{*}$ Multivariate analysis; = regression; CIRS $(\mathrm{G})=$ Cumulative Illness Rating Scale for Geriatrics.

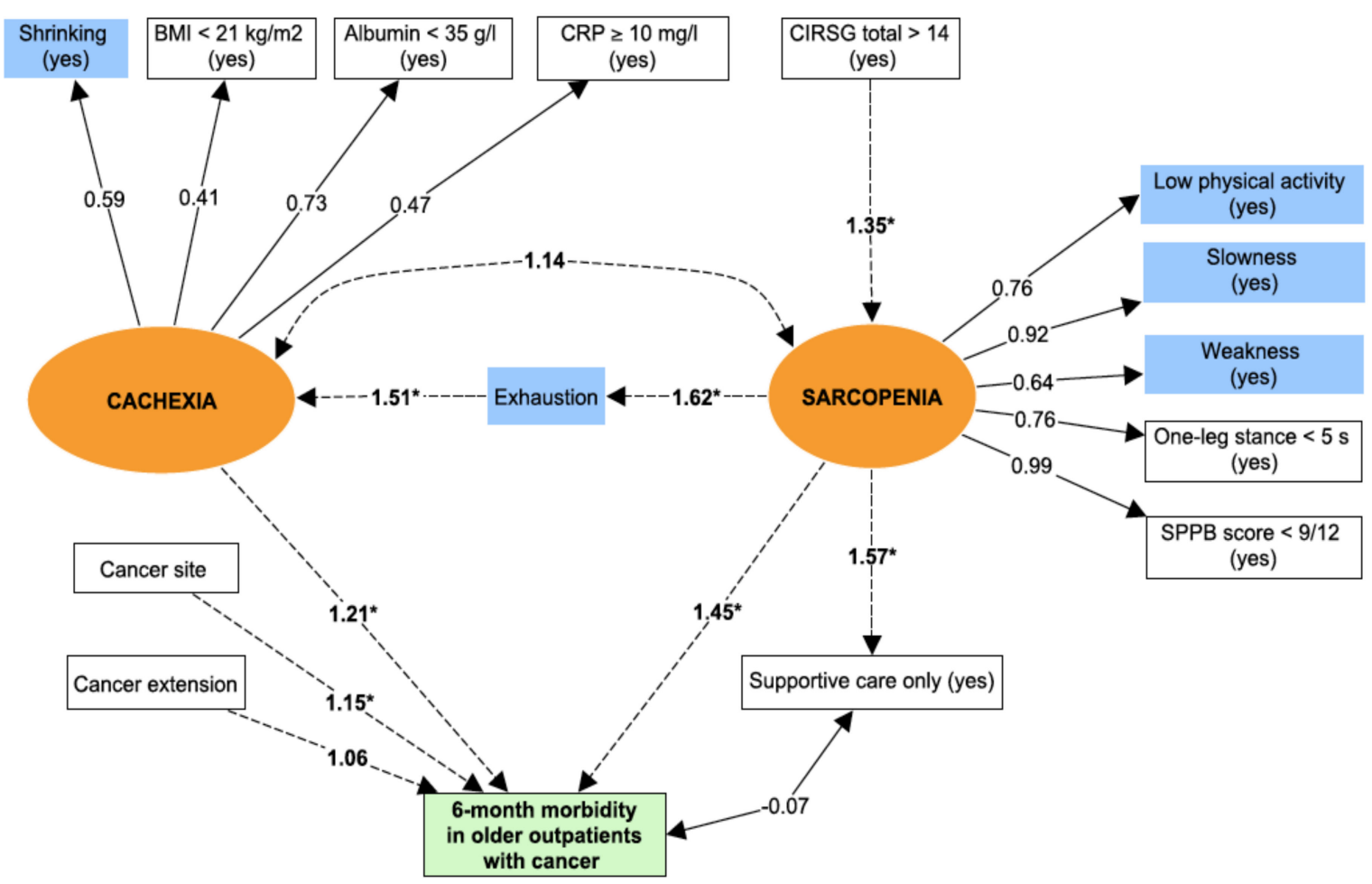

Figure 5. The pathways of the frailty phenotype leading to. Circles (orange) = latent variables; Rectangles = observed variables; Correlations: dotted lines; Regressions: solid lines; Covariance: * $p$ value (for regressions) $<0.05$; Blue rectangle $=$ Frailty criteria; Green rectangle = Outcome. Latent variables represent non-observed variables but which summarize a set of directly observed variables. Latent variables are constructed by using strong correlations between observed variables. Regressions are then used to assess association between latent variables and outcomes. 


\section{Discussion}

We external validated the pathophysiological model of the Fried's frailty phenotype in older patients with cancer. We confirmed the plausibility and the consistency of this model with regard to 6-month morbidity (unplanned hospitalization and/or disability and/or fall) and mortality. The latent variables sarcopenia and cachexia were both directly associated with 6-month mortality and morbidity. Cachexia had the strongest association with mortality, and sarcopenia had the strongest association with morbidity. Exhaustion was found to significantly influence the relationship between sarcopenia and cachexia, and cachexia was found to be the end stage of frailty.

Understanding the pathophysiology of frailty is a key issue in preventing related complications in older patients with cancer. Here, we provided a comprehensive model of the frailty phenotype centred on sarcopenia and cachexia in older patients with cancer. Sarcopenia and cachexia are highly prevalent in cancer patients (particularly in older adults), with a prevalence of between $10 \%$ and $75 \%$ for sarcopenia and between $25 \%$ and $80 \%$ for cachexia (depending on the cancer site/extension) [21,22]. In line with our results, although both sarcopenia and cachexia involve muscle wasting, the former appears to be more strongly related to impaired physical performances, and the latter appears to be more related to malnutrition and inflammation $[5,6]$. However, the two syndromes may be at opposite ends of a continuum (starting with sarcopenia and ending with cachexia) mediated by exhaustion. In line with our findings, exhaustion was found to be significantly associated with sarcopenia in the context of cachexia in two recent observational studies [4,23].

As described above, we found that the comorbidity burden was directly associated with sarcopenia in each of our two models; indeed, comorbidities are well-known risk factors for sarcopenia [5]. However, we found that cancer-related data were not directly associated with the pathophysiology of frailty but were independently associated with mortality and morbidity. Hence, the frailty phenotype does not appear to be influenced by comorbidities such as cancer [3].

Our study had several strengths. First, we longitudinally assessed four frailty-related outcomes (i.e., mortality, unplanned hospitalization, disability, and falls) via regular patient monitoring. Second, the study is the first (to the best of our knowledge) to have externally validated the pathophysiology of frailty in older patients with cancer. Third, our analysis was based on a robust methodology (SEM) that enabled us to understand the nature of the pathological pathways leading to the frailty phenotype.

The main limitation of our study was probably its overestimation of the prevalence of frailty. First, the oncologists, surgeons and other physicians had referred patients who they already suspected to be frail. Moreover, the modified version of the frailty phenotype could lead also to a measurement bias. Another limit is the relative high number of missing data for albumin and CRP could lead to selection and classification biases which were minimized by multiple imputation. At last, although muscle mass was not directly measured, our model remains plausible by summarizing a set of directly observed variables related to physical performances.

Our study confirms the value of using the frailty phenotype to define frailty in older patients with cancer in routine clinical practice; the phenotype is based on a solid pathophysiological model, and can potentially be reversed by target geriatric interventions [24]. In particular, our study results show that sarcopenia and cachexia are risk factors for mortality and morbidity in older cancer patients. Moreover, our model suggests a direct pathway from comorbidities to sarcopenia. It is known that rehabilitation approaches had to be multidisciplinary for improving muscle mass (i.e., dietary, physical activity with resistance training) [25-27]. Our model suggests that it may also be multi-domain by targeting comorbidities as well $[28,29]$. A further validation of our study results would be needed with a longer follow-up.

\section{Conclusions}

We used SEM to confirm the plausibility and the consistency of the pathophysiological model of frailty established by Fried et al. Sarcopenia and cachexia were directly associated with 6-month 
mortality and morbidity in older patients with cancer. Exhaustion was a mediating variable between sarcopenia and cachexia.

Author Contributions: F.P., E.P., and F.C.-P conceived and designed the experiments; F.P. performed the experiments; F.P. and F.C.-P. analyzed the data; M.L., C.M.-T., Y.R. and E.P. contributed analysis; F.P., M.L., C.M.-T, Y.R., E.P. and F.C.P. wrote the paper. All authors have read and agreed to the published version of the manuscript.

Funding: This research received no external funding.

Conflicts of Interest: The authors declare no conflict of interest.

\section{References}

1. Walston, J.; Hadley, E.C.; Ferrucci, L.; Guralnik, J.M.; Newman, A.B.; Studenski, S.A.; Ershler, W.B.; Harris, T.; Fried, L.P. Research Agenda for Frailty in Older Adults: Toward a Better Understanding of Physiology and Etiology: Summary from the American Geriatrics Society/National Institute on Aging Research Conference on Frailty in Older Adults. J. Am. Geriatr. Soc. 2006, 54, 991-1001. [CrossRef] [PubMed]

2. Buta, B.J.; Walston, J.D.; Godino, J.G.; Park, M.; Kalyani, R.R.; Xue, Q.-L.; Bandeen-Roche, K.; Varadhan, R. Frailty assessment instruments: Systematic characterization of the uses and contexts of highly-cited instruments. Ageing Res. Rev. 2016, 26, 53-61. [CrossRef] [PubMed]

3. Fried, L.P.; Tangen, C.M.; Walston, J.; Newman, A.B.; Hirsch, C.; Gottdiener, J.; Seeman, T.; Tracy, R.; Kop, W.J;; Burke, G.; et al. Frailty in older adults: Evidence for a phenotype. J. Gerontol. A Biol. Sci. Med. Sci. 2001, 56, M146-M157. [CrossRef] [PubMed]

4. Tsutsumimoto, K.; Doi, T.; Makizako, H.; Hotta, R.; Nakakubo, S.; Makino, K.; Suzuki, T.; Shimada, H. Aging-related anorexia and its association with disability and frailty. J. Cachexia Sarcopenia Muscle 2018, 9, 834-843. [CrossRef]

5. Cruz-Jentoft, A.J.; Bahat, G.; Bauer, J.; Boirie, Y.; Bruyère, O.; Cederholm, T.; Cooper, C.; Landi, F.; Rolland, Y.; Sayer, A.A.; et al. Sarcopenia: Revised European consensus on definition and diagnosis. Age Ageing 2019, 48, 16-31. [CrossRef]

6. Fearon, K.; Strasser, F.; Anker, S.D.; Bosaeus, I.; Bruera, E.; Fainsinger, R.L.; Jatoi, A.; Loprinzi, C.; MacDonald, N.; Mantovani, G.; et al. Definition and classification of cancer cachexia: An international consensus. Lancet Oncol. 2011, 12, 489-495. [CrossRef]

7. Ethun, C.G.; Bilen, M.A.; Jani, A.B.; Maithel, S.K.; Ogan, K.; Master, V.A. Frailty and cancer: Implications for oncology surgery, medical oncology, and radiation oncology. CA. Cancer J. Clin. 2017, 67, 362-377. [CrossRef]

8. Pamoukdjian, F.; Aparicio, T.; Zebachi, S.; Zelek, L.; Paillaud, E.; Canoui-Poitrine, F. Comparison of Mobility Indices for Predicting Early Death in Older Patients With Cancer: The Physical Frailty in Elderly Cancer Cohort Study. J. Gerontol. A Biol. Sci. Med. Sci. 2020, 75, 189-196. [CrossRef]

9. Miller, M.D.; Paradis, C.F.; Houck, P.R.; Mazumdar, S.; Stack, J.A.; Rifai, A.H.; Mulsant, B.; Reynolds, C.F. Rating chronic medical illness burden in geropsychiatric practice and research: Application of the Cumulative Illness Rating Scale. Psychiatry Res. 1992, 41, 237-248. [CrossRef]

10. Katz, S.; Downs, T.D.; Cash, H.R.; Grotz, R.C. Progress in development of the index of ADL. Gerontologist 1970, 10, 20-30. [CrossRef]

11. Lawton, M.P.; Brody, E.M. Assessment of older people: Self-maintaining and instrumental activities of daily living. Gerontologist 1969, 9, 179-186. [CrossRef] [PubMed]

12. Raynaud-Simon, A.; Revel-Delhom, C.; Hébuterne, X. French Nutrition and Health Program, French Health High Authority Clinical practice guidelines from the French Health High Authority: Nutritional support strategy in protein-energy malnutrition in the elderly. Clin. Nutr. Edinb. Scotl. 2011, 30, 312-319. [CrossRef] [PubMed]

13. Guralnik, J.M.; Simonsick, E.M.; Ferrucci, L.; Glynn, R.J.; Berkman, L.F.; Blazer, D.G.; Scherr, P.A.; Wallace, R.B. A Short Physical Performance Battery Assessing Lower Extremity Function: Association With Self-Reported Disability and Prediction of Mortality and Nursing Home Admission. J. Gerontol. 1994, 49, M85-M94. [CrossRef] [PubMed]

14. Vellas, B.J.; Wayne, S.J.; Romero, L.; Baumgartner, R.N.; Rubenstein, L.Z.; Garry, P.J. One-leg balance is an important predictor of injurious falls in older persons. J. Am. Geriatr. Soc. 1997, 45, 735-738. [CrossRef] [PubMed] 
15. Clément, J.P.; Nassif, R.F.; Léger, J.M.; Marchan, F. Development and contribution to the validation of a brief French version of the Yesavage Geriatric Depression Scale. L'Encéphale 1997, 23, 91-99. [PubMed]

16. Folstein, M.F.; Folstein, S.E.; McHugh, P.R. "Mini-mental state": A practical method for grading the cognitive state of patients for the clinician. J. Psychiatr. Res. 1975, 12, 189-198. [CrossRef]

17. Lahousse, L.; Maes, B.; Ziere, G.; Loth, D.W.; Verlinden, V.J.A.; Zillikens, M.C.; Uitterlinden, A.G.; Rivadeneira, F.; Tiemeier, H.; Franco, O.H.; et al. Adverse outcomes of frailty in the elderly: The Rotterdam Study. Eur. J. Epidemiol. 2014, 29, 419-427. [CrossRef]

18. Avila-Funes, J.A.; Helmer, C.; Amieva, H.; Barberger-Gateau, P.; Goff, M.L.; Ritchie, K.; Portet, F.; Carrière, I.; Tavernier, B.; Gutiérrez-Robledo, L.M.; et al. Frailty among community-dwelling elderly people in france: The three-city study. J. Gerontol. A Biol. Sci. Med. Sci. 2008, 63, 1089. [CrossRef]

19. Shine, B.; de Beer, F.C.; Pepys, M.B. Solid phase radioimmunoassays for human C-reactive protein. Clin. Chim. Acta Int. J. Clin. Chem. 1981, 117, 13-23. [CrossRef]

20. Schreiber, J.B. Core reporting practices in structural equation modeling. Res. Soc. Adm. Pharm. 2008, 4, 83-97. [CrossRef]

21. Ryan, A.M.; Power, D.G.; Daly, L.; Cushen, S.J.; Bhuachalla, Ë.N.; Prado, C.M. Cancer-associated malnutrition, cachexia and sarcopenia: The skeleton in the hospital closet 40 years later. Proc. Nutr. Soc. 2016, 75, 199-211. [CrossRef] [PubMed]

22. Pamoukdjian, F.; Bouillet, T.; Lévy, V.; Soussan, M.; Zelek, L.; Paillaud, E. Prevalence and predictive value of pre-therapeutic sarcopenia in cancer patients: A systematic review. Clin. Nutr. 2018, 37, 1101-1113. [CrossRef] [PubMed]

23. Sur, M.D.; Namm, J.P.; Hemmerich, J.A.; Buschmann, M.M.; Roggin, K.K.; Dale, W. Radiographic Sarcopenia and Self-reported Exhaustion Independently Predict NSQIP Serious Complications After Pancreaticoduodenectomy in Older Adults. Ann. Surg. Oncol. 2015, 22, 3897-3904. [CrossRef] [PubMed]

24. Gill, T.M.; Gahbauer, E.A.; Allore, H.G.; Han, L. Transitions between frailty states among community-living older persons. Arch. Intern. Med. 2006, 166, 418-423. [CrossRef]

25. Marzetti, E.; Calvani, R.; Tosato, M.; Cesari, M.; Di Bari, M.; Cherubini, A.; Broccatelli, M.; Savera, G.; D’Elia, M.; Pahor, M.; et al. Physical activity and exercise as countermeasures to physical frailty and sarcopenia. Aging Clin. Exp. Res. 2017, 29, 35-42. [CrossRef]

26. Minnella, E.M.; Awasthi, R.; Loiselle, S.-E.; Agnihotram, R.V.; Ferri, L.E.; Carli, F. Effect of Exercise and Nutrition Prehabilitation on Functional Capacity in Esophagogastric Cancer Surgery: A Randomized Clinical Trial. JAMA Surg. 2018, 153, 1081-1089. [CrossRef]

27. Davis, M.P.; Panikkar, R. Sarcopenia associated with chemotherapy and targeted agents for cancer therapy. Ann. Palliat. Med. 2018, 8, 86-101. [CrossRef]

28. Deimling, G.T.; Arendt, J.A.; Kypriotakis, G.; Bowman, K.F. Functioning of Older, Long-Term Cancer Survivors: The Role of Cancer and Comorbidities. J. Am. Geriatr. Soc. 2009, 57, S289-S292. [CrossRef]

29. Van der Leeden, M.; Huijsmans, R.J.; Geleijn, E.; de Rooij, M.; Konings, I.R.; Buffart, L.M.; Dekker, J.; Stuiver, M.M. Tailoring exercise interventions to comorbidities and treatment-induced adverse effects in patients with early stage breast cancer undergoing chemotherapy: A framework to support clinical decisions. Disabil. Rehabil. 2018, 40, 486-496. [CrossRef]

(C) 2020 by the authors. Licensee MDPI, Basel, Switzerland. This article is an open access article distributed under the terms and conditions of the Creative Commons Attribution (CC BY) license (http://creativecommons.org/licenses/by/4.0/). 